\title{
Résultats et problèmes posés par l'introduction de Mollusques Zonitidae dans quelques biotopes à Limnées tronquées \\ en Indre et Haute-Vienne
}

\author{
par D. RONDELAUD *
}

Laboratoire de Biologie animale ( $\mathrm{P}^{\mathrm{r}} \mathrm{R}$. SourIE), Faculté des Sciences, F 87060 Limoges, et Laboratoire d'Histologie-Embryologie-Cytogénétique ( $\mathrm{P}^{\mathrm{r}} \mathrm{D}$. BARTHE), Faculté de Médecine, F 87032 Limoges.

\section{Résumé.}

L'introduction systématique de Mollusques Zonitidae: Zonitoides nitidus et Oxychlus draparnaudi, isolément ou en association, sur divers types d'habitats à Lymnaea truncatula en Haute-Vienne (1974-1976) montre:

- que l'élimination des Limnées tronquées est complète au bout de 2 ans dans les habitats temporaires recouverts d'une concentration d'herbes juste fauchées au début de l'assèchement estival. La présence de pierres formant abris accroît les nombres de Limnées survivantes.

- que l'introduction de prédateurs sans fauche préalable n'est efficace que sur les berges de rivières.

\section{Summary.}

Results and problems set by the introduction of Zonitidae snails in some biotopes of Lymnaea truncatula Müller in Indre and Haute-Vienne, France.

Systematic placing of Zonitidea snails: Zonitoides nitidus and Oxychilus draparnaudi, alone or in groups, in different series of habitats of Lymnaea truncatula in Haute-Vienne, France (1974-1976) shows:

- the elimination of Lymnaea truncatula is entire after two years in temporary habitats covered with grass just mowed at the beginning of summer drying. The presence of stones, which make shelters, increases numbers of surviving snails.

- predators-placing without previous grass-mowing is effective only on banks of rivers.

Reçu le 15 février 1977.

(*) Avec l'aide financière de l'Institut de Pathologie Comparée et Expérimentale du Limousin (I.P.C.E.L.). 
Dans une publication antérieure $(1977 \mathrm{~b})$, nous avons défini divers biotopes limousins à Limnées tronquées: prairies marécageuses, jonçaies de pente ou de plateau, zones à empreintes de bovidés, etc... Ils peuvent être caractérisés par des abondances variables de Lymnaea truncatula, l'absence quasi totale de Mollusques prédateurs (Zonitoides nitidus) dans les jonçaies de pente, les variations saisonnières des teneurs en eau des sols et du ruissellement qui conditionnent la vie active des Mollusques.

L'expérimentation en élevage et divers essais sur le terrain (1975, $1976 a$ et $b$, 1977 a) ont montré de plus :

- que l'association de 2 Mollusques Zonitidae complémentaires dans leurs rôles de prédateurs aboutit à une élimination plus efficace des Limnées tronquées que celle résultant de l'utilisation d'un seul prédateur ; milieu ;

- que la période de prédation se situe au début du déssèchement estival du

- que la couverture d'herbes fraîchement fauchées, en entretenant plus longtemps l'humidité résiduelle, favorise la prédation.

Il était utile de vérifier les résultats obtenus dans le cadre d'une enquête plus large dans les milieux naturels et sur une période de 2 à 3 ans. Le travail présent correspond à ce but et regroupe les résultats obtenus ainsi que les problèmes posés par l'emploi de 2 Mollusques Zonitidae: Zonitoides nitidus et Oxychilus draparnaudi. L'introduction de ces deux espèces, soit isolément, soit en association, est pratiquée sur deux types d'habitats :

- avec couverture d'herbe juste fauchée au début de l'assèchement estival ;

- sans modification du milieu naturel à la même époque.

Les données proviennent d'expériences faites de 1974 à 1976 en Haute-Vienne. Quelques essais portent sur des peuplements de Limnées tronquées situés sur des berges de rivière (Ia Creuse à Saint-Gaultier, Indre).

\section{Protocole expérimental}

Les habitats sont choisis au printemps en raison de la densité des Limnées tronquées. Deux méthodes sont utilisées :

a) implantation modérée (ou massive) de prédateurs avec concentration d'herbe fauchée dans les habitats au début de l'assèchement estival.

Vingt prédateurs adultes sont introduits pour un $\mathrm{m}^{2}$ de surface colonisable par les Limnées tronquées, juste lors de la disparition de l'eau stagnante (implantation modérée). Quelques habitats ont fait l'objet d'une introduction massive de prédateurs $\left(50 / \mathrm{m}^{2}\right)$. Dans le cas de l'association Zonitoides-Oxychilus, l'apport de cette dernière se monte à $20 \%$ des individus introduits.

Les relevés ultérieurs ne portent que sur les effectifs de Limnées et de prédateurs retrouvés au cours des années suivantes, juste avant l'application éventuelle d'une concentration d'herbes fauchées sur ces habitats; 
b) implantation massive de prédateurs sans modification du milieu naturel.

Les effectifs de prédateurs introduits par $\mathrm{m}^{2}$ de surface colonisée par les Limnées tronquées s'élèvent à 60 individus (avec $50 \%$ d'Oxychilus lors de l'emploi de l'association Zonitoides-Oxychilus).

Les Mollusques des deux espèces sont mis au jeûne pendant 8 jours et placés sur le terrain avant la disparition de l'eau stagnante. Lorsque apparaît la première fente de rétraction dans le sol, un dénombrement porte sur les Limnées et les prédateurs survivants.

\section{Résultats et interprétations}

\section{$1^{\circ}$ Expérimentation sous le couvert d'herbes fauchées.}

A. - Utilisation de Zonitoides nitidus comme seul prédateur.

Le tableau $I$ résume les résultats recueillis. Nous pouvons en déduire les interprétations suivantes :

- dans les habitats temporaires s'asséchant l'été :

- l'introduction de Zonitoides en quantité modérée $\left(20 / \mathrm{m}^{2}\right)$ entraîne la disparition des Limnées dans $50 \%$ des localités la première année (localités 1 à 10).

- la descendance des prédateurs dans ces localités en 1975 y est faible par rapport aux effectifs initiaux de parents. Les nombres sont cependant suffisants pour permettre l'élimination des Limnées dans 4 localités $(1,4,5$ et 10$)$ lors d'une nouvelle application d'herbes fauchées sur les habitats.

- si des prédateurs sont réintroduits à ce moment-là (localités 11 à 13), les Limnées — déjà réduites en nombre - sont toutes consommées.

- l'emploi de Zonitoides en nombres élevés $\left(50 / \mathrm{m}^{2}\right)$ dans quelques habitats (localités 14 à 17) ne permet pas l'élimination totale des Limnées; cependant la descendance issue de celles qui ont survécu est nettement plus faible en 1975 que celle des autres localités déjà citées.

- dans une dépression marécageuse de pente (localité 18), les résultats sont voisins de ceux obtenus dans les prairies marécageuses: les Limnées sont consommées dès la première année et les effectifs de prédateurs diminuent régulièrement au fur et à mesure des années (en préparation 3).

- dans les habitats permanents - avec niveau d'eau variable —, l'introduction de Zonitoides en quantité modérée (localités 19 à 21) avec concentration d'herbes fauchées sur les berges des habitats entraîne la persistance d'effectifs assez élevés de Limnées et de prédateurs. La répétition de la fauche les années suivantes - associée ou non à la réintroduction de prédateurs (localités 22 et 23) - ne modifie pas les résultats déjà obtenus.

Remarque: dans certaines circonstances, la prédation s'est révélée moins efficace. Nous indiquons ci-dessous les causes : 


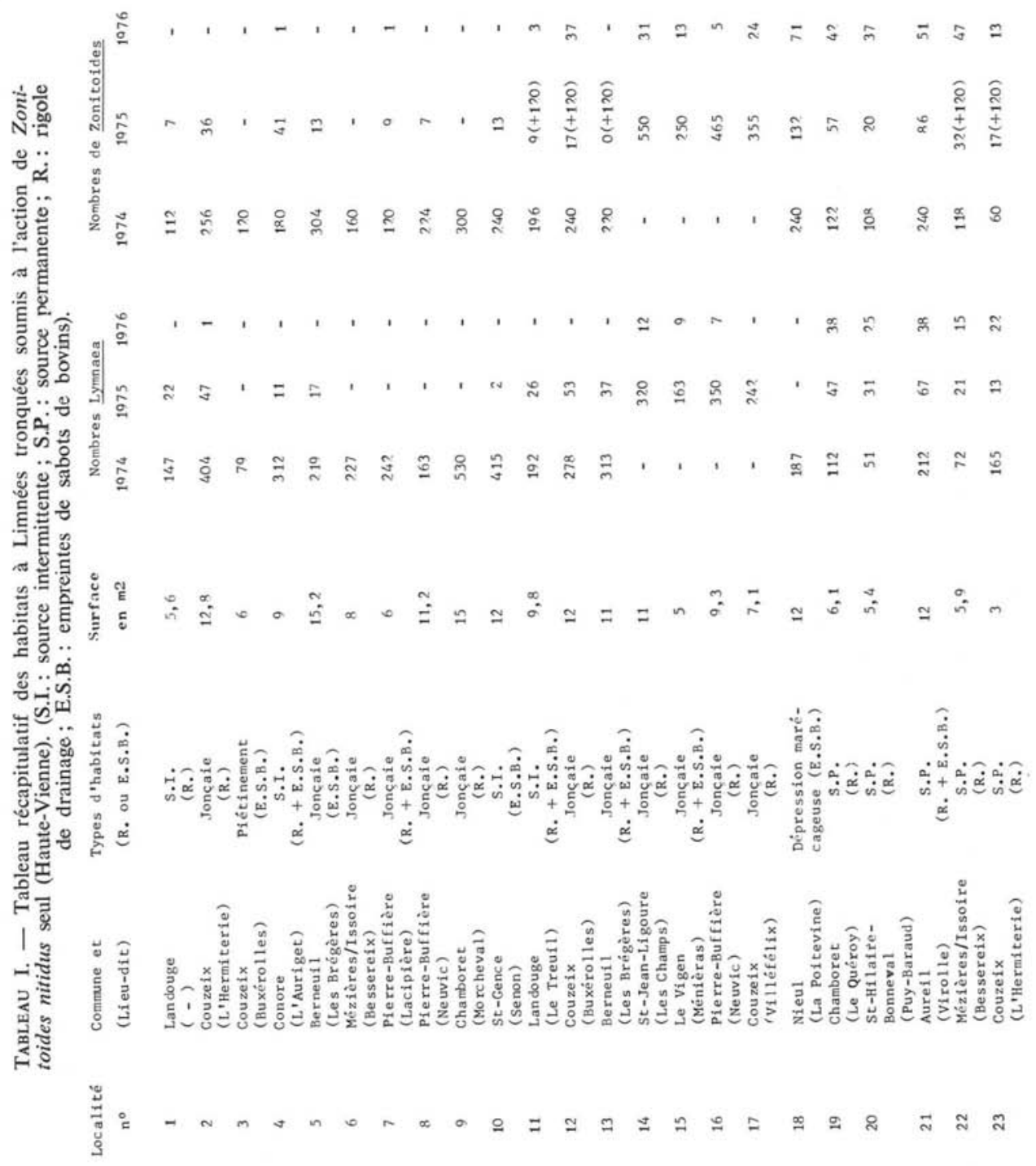


- cas $\mathrm{n}^{\circ} 1$ (4 exemples) : survenue d'une précipitation de forte intensité lors de l'essai. Ceci entraîne le départ des prédateurs de la zone d'expérience et la survie de 25 à $58 \%$ des Limnées.

- cas $\mathrm{n}^{\circ} 2$ (1 exemple): présence d'Acariens phorétiques sur les coquilles des prédateurs. Ces derniers finissent par rester rétractés dans leur coquille avec mort éventuelle.

- cas $\mathrm{n}^{\circ} 3$ ( 1 exemple) : destruction des prédateurs par les passages répétés des ovins dans les rigoles.

B. - L'association Zonitoides nitidus-Oxychilus draparnaudi.

Le tableau II regroupe les résultats obtenus avec cette association sur quelques jonçaies de pente et des berges de rivières. Nous pouvons en tirer les constatations suivantes :

- certaines Limnées subsistent au cours de la deuxième année en certaines localités.

- Zonitoides persiste en nombres réduits dans les jonçaies de pente et en nombres élevés au niveau des berges de rivières.

- les quelques Oxychilus retrouvés la deuxième année se situent tous sous des pierres, à la limite des zones d'essais.

\section{$2^{\circ}$ Expérimentation en milieu naturel non modifié.}

Les essais portent sur des peuplements assez denses de Limnées tronquées vivant:

- sur des jonçaies de pente en Haute-Vienne (séries S I et S III) ;

- sur les berges de la Creuse à Saint-Gaultier, Indre (séries S II et S IV).

Les divers résultats sont rassemblés dans la figure 1. Nous pouvons en dégager les conclusions suivantes :

— dans les jonçaies de pente (fig. $1 \mathrm{~A}$ ), l'assèchement est souvent très rapide (3-4 jours). L'introduction de Zonitoides avant la disparition de l'eau de ruissellement

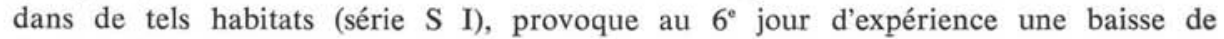
$64 \%(55-73 \%)$ des effectifs de Limnées par rapport aux nombres initiaux.

Pour l'association Zonitoides-Oxychilus (série S III), les nombres de Limnées consommées durant la même période atteignent $73 \%$ (69-79\%).

La rapidité de l'assèchement estival de ce type d'habitat limite la durée de prédation des Limnées (Rondelaud et Morel-Vareille, 1975), ce qui réduit l'efficacité de cette technique ;

- sur les berges de rivières (fig. 1 A), l'assèchement plus lent (10-12 jours) entraîne une consommation accrue des Limnées tronquées. Les résultats obtenus sont voisins de ceux provenant des jonçaies de pente: ainsi, les nombres de Limnées consommées atteignent $85 \%(81-92 \%)$ dans le cas de Zonitoides utilisé seul (série S II) et $94 \%(92-100 \%)$ dans le cas de l'association Zonitoides-Oxychilus (série S IV) ; 


\begin{tabular}{|c|c|c|c|c|c|c|c|c|c|}
\hline 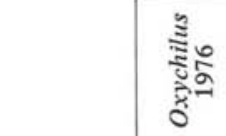 & 0 & m & - & 0 & 0 & - & 0 & $n$ & $=$ \\
\hline 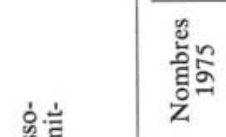 & f & હల & \& & $\stackrel{\sim}{\sim}$ & $尺$ & $\stackrel{\infty}{\sim}$ & $\bar{\lambda}$ & $\underset{\sim}{ }$ & \& \\
\hline \multirow{9}{*}{ 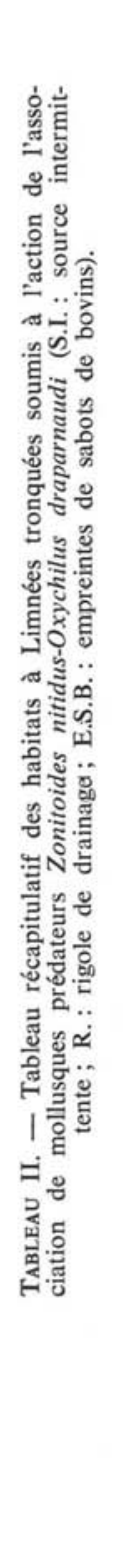 } & ส & $\bar{m}$ & $\tilde{\imath}$ & 0 & $m$ & 0 & $\bar{m}$ & $\stackrel{\infty}{\forall}$ & $\hat{3}$ \\
\hline & $\stackrel{0}{\circ}$ & త్ & $\stackrel{\circ}{\sim}$ & $\cong$ & $\underset{\sim}{\mathbb{~}}$ & 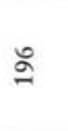 & $\ddot{\infty}$ & ฉ & \& \\
\hline & 0 & $\simeq$ & $r$ & 0 & 0 & 0 & - & 0 & 0 \\
\hline & $\frac{0}{7}$ & ๙ู & $\frac{m}{\lambda}$ & $\vec{\lambda}$ & 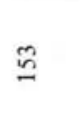 & $\frac{n}{m}$ & $\cong$ & $\stackrel{\sim}{\pi}$ & $\stackrel{\infty}{\infty}$ \\
\hline & $\stackrel{\overbrace{}}{\varrho}$ & $\approx$ & $\stackrel{\infty}{\circ}$ & $\stackrel{\sim}{\sim}$ & $\stackrel{\infty}{=}$ & $\simeq$ & in & 0 & $a$ \\
\hline & 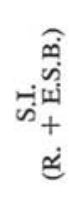 & ต่อิ & 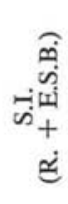 & ต่ं & 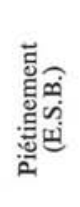 & ம் & 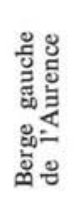 & 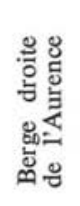 & 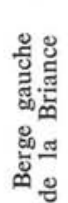 \\
\hline & 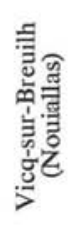 & 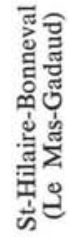 & 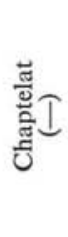 & 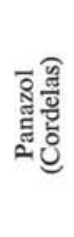 & 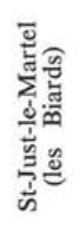 & 默 & 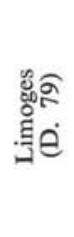 & 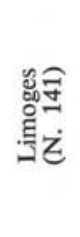 & 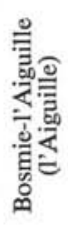 \\
\hline & $\begin{array}{l}\vdots \\
\vdots \\
\vdots\end{array}$ & $\begin{array}{l}\vdots \\
\vdots \\
\vdots\end{array}$ & $\begin{array}{l}\vdots \\
\vdots\end{array}$ & $\begin{array}{c}\vdots \\
\vdots \\
\vdots\end{array}$ & $\begin{array}{c}\vdots \\
\vdots \\
\vdots\end{array}$ & $\begin{array}{c}\vdots \\
\vdots \\
\vdots\end{array}$ & $\begin{array}{l}\vdots \\
\vdots \\
\vdots\end{array}$ & $\begin{array}{l}\vdots \\
\vdots \\
\vdots\end{array}$ & $\begin{array}{c}\vdots \\
\vdots\end{array}$ \\
\hline & - & $\mathrm{N}$ & $m$ & $\nabla$ & $n$ & 0 & $n$ & $\infty$ & $a$ \\
\hline
\end{tabular}



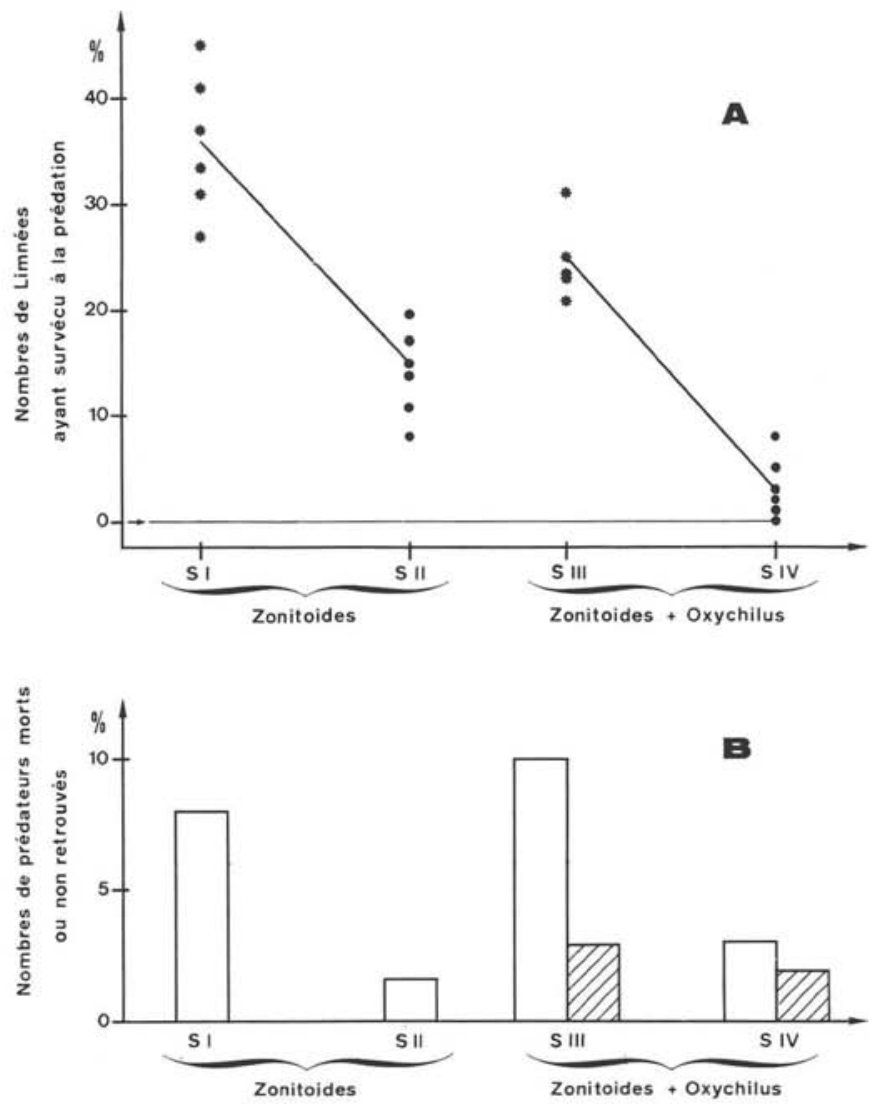

FIG. 1. - Résultats concernant l'action de Zonitoides seul ou de l'association ZonitoidesOxychilus sur des peuplements de Limnées tronquées vivant:

- sur des jonçaies de pente en Haute-Vienne (séries S I et S III).

- sur les berges de la Creuse à Saint-Gaultier, Indre (séries SII et S IV).

A : les nombres de Limnées tronquées survivantes recueillies à la fin des expériences. B : les nombres de prédateurs morts ou non retrouvés (Oxychilus : en hachuré).

- les nombres de Zonitoides (fig. 1 B) morts ou non retrouvés à la fin des essais varient selon le type d'habitat. Les chiffres concernant Oxychilus draparnaudi restent faibles et constants dans les deux cas.

La survenance de précipitations lors des essais (3 cas) retarde temporairement l'action des prédateurs tont en prolongeant l'humidité des habitats en voie d'assèchement. Les pertes de prédateurs sont légèrement plus élevées. 


\section{Discussion}

Les résultats présentés ci-dessus nous permettent de dégager les points suivants:

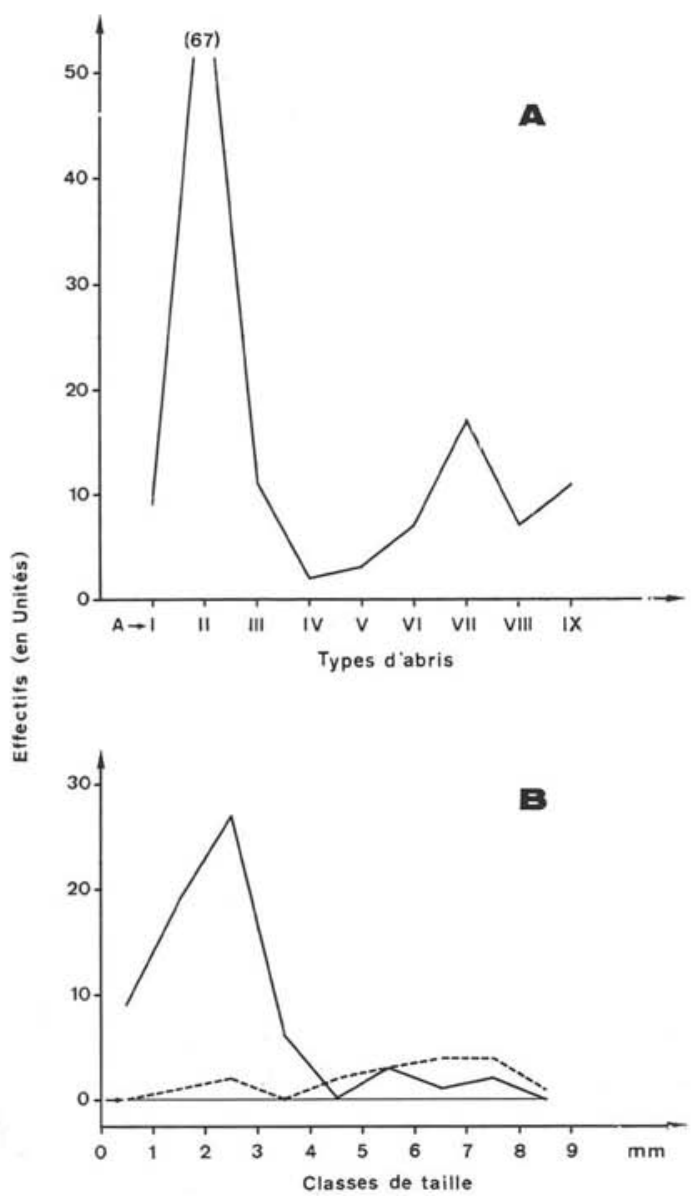

FIG. 2. - La distribution des Limnées tronquées recueillies à la fin de l'estivation (localités soumises à une expérimentation sous le couvert d'herbes fauchées) :

- en fonction du type d'abri (A I : base de drains ; A II : pierres, A III : parpaings ; A IV : briques ; A V: planches; A VI: base de plantes; A VII : accrochage aux plantes; A VIII : sur le sol; A IX : cause non retrouvée),

- en fonction de leur hauteur (Limnées retrouvées sous les pierres: traits continus; Limnées accrochées aux plantes: traits pointillés). a) Les nombres de Limnées tronquées ayant survécu à la prédation à l'issue de la première année sont plus élevés dans les conditions naturelles que lors des expériences préliminaires (1975, $1976 a$ ). Il est nécessaire d'en rechercher l'explication. La figure 2 indique la distribution des Limnées tronquées retrouvées dans 37 localités ayant fait l'objet d'une expérimentation: les Mollusques sont recherchés et comptés lors des premières pluies précédant la remise en eau des rigoles. Il apparaît alors que les survivantes ont trouvé des abris de divers types (fig. $2 \mathrm{~A}$ ) et qu'il s'agit surtout de jeunes individus :

- 67 Limnées survivantes ont été retrouvées sous des pierres - de dimensions supérieures à $8 \mathrm{~cm}-$ (fig. $2 \mathrm{~A}$ ). Ce sont surtout de jeunes individus $(1-3 \mathrm{~mm}$ : fig. $2 \mathrm{~B})$;

- 17 Mollusques ont été observés sur des plantes à $2 \mathrm{~cm}$ au-dessus du sol (fig. $2 \mathrm{~A}$ ). Mais nous en avons compté aussi 28 mortes dans leur coquille;

- les autres types d'abris n'ont montré qu'un faible nombre de Limnées survivantes.

La présence de pierres plus ou moins nombreuses sur l'habitat influe donc sur la survie des Limnées lors de l'application d'une technique de lutte biologique par prédation. 
b) En Haute-Vienne, l'élimination des Limnées tronquées est complète au bout de 2 années dans la quasi-totalité des habitats temporaires avec concentration d'herbes juste fauchées au début de l'assèchement estival. La descendance des prédateurs introduits est peu nombreuse. Par contre, l'introduction de Zonitoides dans les habitats permanents limite seulement l'évolution démographique des Limnées.

c) L'association de Mollusques: Zonitoides nitidus-Oxychilus draparnaudi utilisée systématiquement sur le terrain est plus efficace que Zonitoides employé seul. Les résultats sont donc conformes à ceux fournis par les essais préliminaires (1976 $a$, $1977 a)$.

d) L'introduction d'effectifs élevés de prédateurs dans des habitats à Limnées tronquées sans modification du milieu naturel doit être réservée aux berges de rivière : en effet, ces dernières se déssèchent lentement et l'action des prédateurs est maximale. Cette méthode présente l'avantage d'éviter une fauche de la végétation hygrophile.

Par contre, les jonçaies de pente et les empreintes de sabots de bovins en HauteVienne doivent faire l'objet d'une introduction de Mollusques Zonitidae avec concentration d'herbes juste fauchées sur ces habitats au début de l'assèchement estival.

En conclusion, l'emploi de ces deux méthodes de lutte biologique par prédation permet donc un contrôle efficace des populations de Limnées tronquées en Indre et Haute-Vienne. Il conviendra cependant d'en préciser les effets à long terme.

\section{Bibliographie}

Rondelaud (D.), 1975. - La prédation de Lymnaea (Galba) truncatula Müller par Zonitoides nitidus Müller, moyen de lutte biologique. Ann. Parasitol. hum. comp., 50, 55-61.

Rondelaud (D.), $1976 a$. - L'intérêt d'une association de Mollusques Zonitidae dans le contrôle biologique de Lymnaea (Galba) truncatula Müller sur terrains siliceux et calcaires. Ann. Parasitol. hum. comp., 51, 41-49.

Rondelaud (D.), $1976 \mathrm{~b}$. - Analyse biocénotique et activité prédatrice des Carabiques dans les habitats de Lymnaea (Galba) truncatula Müller dans le nord-ouest du Limousin. Bull. Mus. natn. Hist. Nat,, 385, 57-66.

Rondelaud (D.), $1977 a$. - Les aptitudes malacophages de quelques Mollusques Zonitidae et leur intérêt dans le contrôle biologique de Lymnaea (Galba) truncatula Müller. Ann. Parasitol. hum. comp., 52, 411-420.

Rondelaud (D.), 1977 b. - L'évolution démographique de Lymnaea (Galba) truncatula Müller en Haute-Vienne. A propos de 4 années d'observations. Ann. Parasitol. hum. comp., 52, 511-520. 
Rondelaud (D.), 1977. - Les effets à long terme d'un contrôle biologique par prédation. Etude expérimentale du devenir de quelques populations de Mollusques. Ann. Parasitol. hum. comp. (à paraître).

Rondelaud (D.) et Morel-Vareille (C.), 1975. - Distribution estivale et survie des Limnées tronquées Lymnaea (Galba) truncatula Müller saines ou infestées par Fasciola hepatica L. Ann. Parasitol. hum. comp., 50, 603-616. 